\title{
LA EDUCACIÓN SUPERIOR Y EL PROCESO DE TRANSFORMACIÓN SOCIAL EN EL ECUADOR
}

\author{
HIGHER EDUCATION AND SOCIAL TRANSFORMATION PROCESS IN ECUADOR \\ LaUra AMarilis Borja Herrera * \\ Ena Maritza Feijoo González ** \\ Néstor Daniel Gutierréz Jaramillo *** \\ Raquel Magali Jaramillo Simbaña **** \\ Milca NaAra Orellana Ulloa ***** \\ Docentes de la Universidad Técnica de Machala - Ecuador \\ Asesora: BeAtriz Herrera García \\ Docente Principal de la Facultad de Ciencias Contables - UNMSM
}

[Recepción: Setiembre de 2014/ Conformidad: Octubre 2014]

\section{RESUMEN}

La presente investigación se realiza a partir del nacimiento del Ecuador como República Independiente; y, el análisis sobre el recorrido de la educación superior ecuatoriana, que se inicia con la promulgación de la Primera Constitución Política del 23 de septiembre de 1830 , hasta su última vigente desde el 28 de septiembre de 2008, representando un total de veinte, que han servido de base para este estudio.

Con ello, se ha sintetizado la información en seis fases, las mismas que no han sido determinadas con un rango específico de tiempo, sino en base a la expectativa que generaron los marcos constitucionales que permitieron pasar de un Estado a otro, identificando en cada una de ellas, el lapso de análisis, las acciones más sobresalientes que nos permitan entender y relacionar históricamente el proceso de transformación de la educación superior ecuatoriana que se está viviendo en este momento, en donde todas las instituciones del sistema están sometidas a planes de mejora o fortalecimiento, como parte de la política nacional del Buen Vivir y transformación social proclamada en la nueva Carta Magna vigente. Con esta información, se llega a las respectivas conclusiones.

Palabras clave:

Educación superior; proceso de transformación social.

\begin{abstract}
The present investigation was carried out from birth as an Independent Republic Ecuador; and the analysis on the course of the Ecuadorian higher education, beginning with the promulgation of the First Constitution of September 23, 1830, until his last force since September 28, 2008, representing a total of twenty, which they have provided the basis for this study. With this, the information has been synthesized in six phases, they have not been determined with a specific range of time, but based on the expectation that generated the constitutional framework that allowed to move from one state to another, identifying each of them, the time of analysis, the most outstanding actions that allow us to understand and historically relate the process of transformation of the Ecuadorian higher education that is living in the moment, where all institutions of the system are subject to improvement plans or strengthening, as part of the national policy of Good Living and social transformation proclaimed in the new Constitution in force. With this information, you will reach the respective conclusions.
\end{abstract}

\section{Keywords:}

Higher education; process of social transformation.

\footnotetext{
* Magíster en Docencia Universitaria Investigación Educativa. Email : lborja@utmachala.edu.ec

** Magíster en Tributación y Finanzas. Email : efeijoo@utmachala.edu.ec

*** Magíster en Administración de Empresas. Email : ngutierrez@utmachala.edu.ec

**** Magíster en Docencia y Gerencia en Educación Superior. Email : rmjaramillo@utmachala.edu.ec

*****Magíster en Auditoría y Contabilidad. Email:morellana@utmachala.edu.ec

****** Doctora en Ciencias Económicas. Email : bherrerag@unmsm.edu.pe
} 
Laura Amarilis Borja Herrera - Ena Maritza Feijoo González

Néstor Daniel Gutierréz Jaramillo - Raquel Magali Jaramillo Simbaña

Milca NaAra Orellana Ulloa - Beatriz Herrera García

\section{INTRODUCCIÓN}

La educación superior del Ecuador ha recorrido seis momentos importantes, a partir del reconocimiento del país como república independiente de la Gran Colombia ${ }^{1}$ en 1830, y por espacio de 67 años (1830-1897), se mantuvo la influencia de la iglesia católica, al ser parte de la educación general, presentándose por lo tanto en la segunda fase, la figura de apadrinamiento desde Europa hacia los países, que como Ecuador representaron "sus protegidos culturales".

Este camino recorrido atraviesa verdaderos momentos históricos como el reconocimiento de la educación laica en todos sus niveles, la independencia estructural de la educación superior de los otros niveles educativos; la lucha por la autonomía universitaria liderada por los sectores sociales en busca de los espacios que se merecía la educación superior, sin directrices claras y cuando las hubo, sin la voluntad de tomar decisiones que permitan el mejoramiento del sector; por el contrario, el sistema refleja históricamente un momento de verdadera expansión irresponsable.

Ha sido necesario hacer este análisis para poder entender el rol de la educación superior ecuatoriana en el contexto del momento actual, que en los últimos cinco años ha sido partícipe del cierre de universidades e institutos superiores técnicos y tecnológicos que no han cumplido con la finalidad para la cual fueron creados.

Para el efecto, se hace el análisis de cada uno de estos momentos o fases históricas a través de las seis (06) fases planteadas en el desarrollo del artículo.

\section{PRIMERA FASE:}

\section{LA EDUCACIÓN SUPERIOR CON EL INICIO DE LA VIDA REPUBLICANA}

Con el advenimiento de la vida republicana en el Ecuador y el reconocimiento de la sociedad como "nación", como parte de un proceso que se introdujo en los diferentes países americanos ante la corriente presentada en Europa con respecto a la ruptura de un régimen que no respondía a criterios de libertad y justicia, bajo el apadrinamiento del modelo llamado liberalismo europeo ${ }^{2}$ imperante a principios del siglo XIX, como Estado independiente al amparo de la primera Constitución promulgada en Riobamba el 23 de septiembre de 1830, se consagró como obligación del Estado promover y fomentar la educación pública como elemento prioritario de desarrollo del país, buscando a través de las políticas educativas un rol integrador en busca del mejoramiento de las condiciones de vida de la sociedad e incluso del fomento de las actividades económicas.

En esta fase identificada en un periodo de 22 años, en los cuales la falta de seguridad en el manejo del Estado, evidenciada a través de las siguientes (04) cuatro constituciones aprobadas en este periodo, bajo las influencias externas predominantes en la época, especialmente, con predominio de los modelos europeos, demuestran que la educación en general en su contexto, se desarrollaba en el marco de un modelo pedagógico que representa formas particulares de interrelación entre los parámetros pedagógicos, con lo cual se busca definir la concepción del ser humano que se aspira formar como ideal de persona educada en una determinada sociedad.

Así entonces, en este período la educación superior con una inexistente figura legal independiente, se enmarcó en el modelo pedagógico tradicional ${ }^{3}$, basado en la enseñanza formal, gramatical y literaria.

Un primer intento de cambiar esta estructura que podría ser identificada como una acción significativa es la promulgación de un Decreto en el mes de febrero de 1836, en la presidencia de Vicente Rocafuerte, en donde se expide el Decreto Orgánico de Enseñanza Pública en el que se introdujeron varias reformas a la enseñanza tanto secundaria como superior; pues, la primaria todavía estaba sujeta a la iniciativa y el control privado, de allí que el analfabetismo era muy acentuado.

1 Existió jurídicamente entre 1821 y 1831 y se configuró a partir de la unión de las entidades coloniales del Virreinato de la Nueva Granada, Capitanía General de Venezuela, Presidencia de Quito y la Provincia Libre de Guayaquil.

2 Doctrina que en Europa tuvo su apogeo durante la revolución francesa y que destaca la importancia de los mercados libres y las libertades civiles.

3 Modelo que concibe la enseñanza como un verdadero arte y al profesor como un artesano, donde su función es explicar claramente y exponer de manera progresiva sus conocimientos, enfocándose de manera central en el aprendizaje del alumno.

188/ QVIPURAMAYOC | Vol. 22(42) 2014 
En lo que respecta a la presencia de la religión en la educación, no tuvo la característica de una imposición, sino que seguía los patrones previamente establecidos como es el caso de la única universidad existente en este periodo que fue la Universidad Central de Quito, que bajo el amparo de la Gran Colombia, a la cual pertenecía el Ecuador en ese entonces, se crea el 18 de marzo de 1826 , y que surgió para reemplazar a la Universidad Santo Tomás de Aquino, que pertenecía a los frailes dominicos.

Así entonces, en esta etapa ha sido difícil encontrar evidencias que permitan realizar un análisis particular de la situación de la educación superior en el contexto de la sociedad ecuatoriana; porque siendo parte de un todo, con directrices que no marcaban efectivamente una independencia de factores externos, las propias estructuras la asumían como una prolongación o extensión de la hasta ese entonces, educación secundaria.

\section{SEGUNDA FASE: \\ INFLUENCIA DE LA RELIGIÓN CATÓLICA EN LA EDUCACIÓN}

Esta segunda fase nace con la expedición de la Constitución del 6 de diciembre de 1852, promulgada en Guayaquil, es con la venia de esta Constitución, cuando la iglesia católica asume la más grande influencia en la educación en el Ecuador, lo cual incluso se puso de manifiesto con la ejecución de la primera Reforma Educativa, que por su identificación con la iglesia católica, permitió el ingreso al país de comunidades religiosas, entregándole a los jesuitas la responsabilidad de la enseñanza secundaria, con influencia en el nivel superior que no gozaba de ninguna independencia de gestión, aunque el inventario alcanzó a cuatro universidades públicas: una en la costa y tres, en la sierra.

La evolución del marco constitucional en este periodo igualmente está identificado por una inestabilidad jurídica por cuanto en un periodo de 45 años, se promulgaron cuatro nuevas constituciones, esta etapa históricamente ha sido reconocida como la del "Garcianismo"4 por la influencia del pensamiento de
Gabriel García Moreno, líder político en aquella época, quien al llegar a la presidencia se propuso transformar y mejorar el sistema educativo ecuatoriano, justificando de esta manera la adopción de modelos educativos desarrollados en Europa; especialmente, por las comunidades religiosas, lo que en su momento fue reconocido como beneficioso; puesto que, es en este periodo en donde se inicia el reconocimiento de la necesidad de invertir en educación, lo que estuvo representada en la construcción de escuelas, colegios y material necesario para la labor educativa, pero todo bajo el direccionamiento de las comunidades religiosas posicionadas en el país, al amparo de la Constitución de esa época.

Pese al poder ejercido, García Moreno no pudo controlar la promulgación de la Ley Orgánica de Instrucción Pública en 1863, la que introdujo innovaciones como la estructuración del Consejo General de Instrucción Pública, en donde de los seis integrantes, dos pertenecían a las facultades universitarias y con ello, se pudo señalar que se fijaron las bases para la autonomía universitaria.

El Consejo así estructurado ejerció la supervigilancia y control de la enseñanza en todos sus niveles o grados, determinando además como atribuciones del mismo, normar que la provisión de cátedras debía hacerse por concursos, a través de estrictas pruebas.

\section{TERCERA FASE:}

\section{EL RECONOCIMIENTO DE LA EDUCACIÓN LAICA EN EL ECUADOR Y LA DEFENSA DE LA AUTONOMÍA UNIVERSITARIA}

Con las medidas tomadas al cierre del periodo anterior, el Ecuador se encaminó a un proceso de transformación, considerado como una de las etapas más importantes en su historia de país como república independiente, posterior a la revolución liberal del 5 de junio de 1895 .

Por ello, podría identificarse a esta fase, como de la "lucha por la autonomía de la universidad ecuatoriana", la que parte de la promulgación de la Constitución del 14 de enero de 1897 y que abarcó un periodo de 70 años, en el cual se promulgaron

\footnotetext{
4 El Garcianismo se llama al período que va desde el 1859 al 1875, comprende las presidencias de Gabriel García Moreno, que dio un cierto poder a la Iglesia Católica de su país (Ecuador).
} 
cinco nuevas Constituciones 5 , las que abrieron el espacio para independizar a la educación superior de los otros niveles educativos del Ecuador, a través de la expedición de seis Leyes de Educación Superior entre el 6 de octubre de 1925 y el 6 de junio de 1966, a las cuales se sometieron las 10 universidades existentes en este periodo: 7 públicas y 3 cofinanciadas.

Se señaló como uno de los aspectos trascendentales en esta fase, el nacimiento de la educación laica en el Ecuador, al marcar la independencia de la educación ligada a la religión, dando lugar a una nueva orientación pedagógica basada fundamentalmente, en valores cívicos de la democracia y del liberalismo.

El 6 de octubre de 1918, se expide la primera Ley de Educación Superior del Ecuador y para 1919 por la necesidad de luchar por sus derechos; por primera vez, deciden organizarse los estudiantes universitarios, para unidos emprender un largo camino por el reconocimiento de una auténtica y liberadora educación superior. Para 1925 se expide una nueva Ley de Educación Superior, la misma que históricamente representa el nacimiento de la autonomía universitaria, aunque sus limitaciones permitieron el afianzamiento de la problemática Estado-Universidad-Estado; pues, pese a la aceptación del derecho a la autonomía, debido a algunas potestades de decisión que le eran conferidas al Ministro de Educación, se ponía en riesgo la propia subsistencia de la autonomía; y, es aquí donde la participación de la juventud marcó el paso de la historia universitaria, con acciones de rebeldía surgidas en las propias aulas universitarias, con enfrentamientos constantes que contribuyeron más tarde en 1938, que se consiguiera la ratificación del derecho a la autonomía universitaria con la promulgación de la nueva Ley de Educación Superior en la cual se ratificaron las bases de la autonomía universitaria sustentadas en la Ley de 1925; pero, la lucha se mantenía porque no era suficiente que el derecho estuviera amparado en una Ley, se buscaba que este derecho conste en la Carta Política del Estado, siendo un primer logro el nacimiento oficial de la Federación de Estudiantes Universitarios del Ecuador (FEUE), institución integrada por estudiantes universitarios de todo el país, que asumió como uno de sus principios fundamentales el reconocimiento constitucional de la autonomía universitaria.

Es en la Constitución del 6 de marzo de 1945, en la cual en el artículo 143 se declara que la "Educación constituye función del Estado"; y, se declara además la independencia de la educación superior del resto del sistema.

En los dieciocho años siguientes hasta 1963, la historia no registra acciones relevantes en el desarrollo de la Educación Superior del Ecuador; pero, para este año (1963) cuando se rompe la vida democrática con la presencia de un Gobierno de facto se pone en peligro la autonomía universitaria, la cual fue restituida con la promulgación de la Ley de Educación Superior, expedida el 6 de junio de 1966, mediante decreto 394 del Presidente Interino de la República Clemente Yerovi Indaburu, la que surge a partir de una propuesta de la comunidad universitaria representada por los rectores de las universidades existentes en esa fecha. En esta Ley se reconoce legalmente este derecho para la Educación Superior, cuando en su artículo 2, consagra la autonomía universitaria; y, en el artículo 3 prohíbe al poder ejecutivo de modo terminante, clausurar, reorganizar, disminuir su rentas, retardar su entrega o adoptar medida alguna que menos cabe el funcionamiento normal de tales establecimientos o que viole su libertad o autonomía.

Además, esta Ley excluye al Ministro de Educación como Autoridad Universitaria y otorga la representación estudiantil al Honorable Consejo Universitario de un representante por cada facultad y establece para los órganos de gobierno estudiantil una proporción igual a la mitad del número de profesores que lo entregan. Por lo tanto, esta Ley ha sido considerada como la Ley que consagra real y definitivamente la autonomía de la universidad ecuatoriana.

Como otro de los aspectos relevantes de esta fase y al amparo de la Ley de 1966, que en su Capítulo Segundo, artículo 5 señala que "Para orientar y coordinar la acción de los establecimientos de educación superior y armonizar sus planes pedagógicos, culturales y científicos, se establece el Consejo Nacional

5 Constituciones del Ecuador promulgadas en las siguientes fechas: 22 de diciembre de 1906; 26 de marzo de 1928; 2 de diciembre de 1938; 6 de marzo de 1945 y 31 de diciembre de 1946.

190/ QVIPURAMAYOC | Vol. 22(42) 2014 
de Educación Superior", en cumplimiento de esta disposición legal el Ministro de Educación Pública de ese entonces, convocó a los rectores de las universidades existentes en la Universidad Central del Ecuador, con la presencia de siete rectores de universidades públicas y dos particulares, se constituyó este primer Consejo Nacional de Educación Superior.

Desde nuestro punto de vista, esta fase está representada por el despertar no sólo de la juventud sino de la sociedad en general, en busca de sus derechos, derechos que no fueran suficientes que estuvieran amparados en una Ley, sino que necesitaban un espacio en la carta Suprema de un país que en 1830 proclamó su independencia, pero que no lograba alcanzar la misma 136 años después.

\section{CUARTA FASE:}

\section{SURGIMIENTO DE LA FIGURA LEGAL DEL CONSEJO NACIONAL DE EDUCACIÓN SUPERIOR}

Luego de la fase vivida en el Ecuador, en donde el reconocimiento de los derechos democráticos amparados en la Constitución representaron los aspectos de mayor incidencia social sobre todo en la juventud universitaria; en el Ecuador, se apertura un nuevo período que se inicia en mayo de 1967 con la promulgación de una nueva Constitución, a la que le sucedió otra más, lo que evidencia una mayor estabilidad en el marco constitucional del país en estos 21 años, en los cuales se expidió la séptima Ley de Educación Superior en enero de 1971.

La característica de la educación en general en este periodo, se pone de manifiesto con el reconocimiento del derecho a la educación de los campesinos e indígenas, además se reconoce a la educación especial y técnica, la alfabetización, el suministro de útiles y servicios, la ayuda a la educación particular.

En lo que respecta a la educación superior representada en este periodo por cuarenta y tres (43) universidades, de las cuales veinte (20) eran públicas, doce (12) cofinanciadas y once autofinanciadas, la Constitución de 1967 ratifica lo promulgado constitucionalmente en 1945, con respecto a la autonomía universitaria. Así mismo se ratifican los aspectos relacionados a la obligatoriedad de la educación en el nivel primario y en el ciclo básico del nivel medio, ratificando su gratuidad en los niveles anotados cuando la misma sea impartida en establecimientos oficiales. Para la fecha no se reconocía la gratuidad en la educación superior aún.

Con la nueva Ley de Educación Superior de enero de 1971, en el Gobierno Dictatorial de Velasco Ibarra, se limita el concepto de autonomía universitaria a la "facultad de organizarse, de enseñar y de expedir certificados de estudios" y promueve la figura de un Consejo Nacional de Educación Superior, a quien la Ley le confiere la tarea de dirigir el funcionamiento, unificar el régimen interior, aprobar planes de estudios y programas; etc., pero asimismo se le confiere la facultad para clausurar temporal o definitivamente, las universidades llegando incluso a señalar que era incompatible ser miembro del Consejo con el ejercicio de una cátedra universitaria o politécnica, pero se reconocía la compatibilidad con el desempeño de otra función pública o privada, con estas consideraciones este Consejo fue calificado como sumiso a los intereses de la dictadura; asimismo, la Ley así estructurada fue calificada por la comunidad universitaria como "la carta de la esclavitud", promoviendo por lo tanto un rechazo a la misma.

Pero quizá lo que ocasionó los mayores cuestionamientos fue la decisión de replantear el examen de ingreso considerado como una conquista alcanzada hasta ese momento por la juventud ecuatoriana.

Así entonces el periodo entre 1967 a 1998, es considerado el de la desestabilización política al contar el país con 7 presidentes constitucionales, 1 presidente interino y 2 gobiernos de facto, lo que menos importó en ese lapso fue el estado de la educación superior; por lo tanto, en el ámbito pedagógico la universidad ecuatoriana se continuó desarrollando sobre la base del Modelo Educativo Tradicionalista, que respondía a las exigencias neoliberales de la época.

Este periodo se cierra teniendo vigente la Ley de Universidades y Escuelas Politécnicas expedida el 11 de mayo de 1982 y además con el referente que para 1988 la población estudiantil universitaria alcanzaba la cifra de 195.000 estudiantes de universidades y escuelas politécnicas oficiales y cofinanciadas en el Ecuador (Ramirez \& Analía, 2009). 
Laura Amarilis Borja Herrera - Ena Maritza Feijoo González

Néstor Daniel Gutierréz Jaramillo - Raquel Magali Jaramillo Simbaña

Milca NaAra Orellana Ulloa - Beatriz Herrera García

\section{QUINTA FASE:}

LA INSERCIÓN DE LOS INSTITUTOS TÉCNICOSYTECNOLÓGICOS COMO PARTE DE LA EDUCACIÓN SUPERIOR

Esta quinta fase se origina con la expedición de la Constitución Política del Ecuador publicada el 11 de agosto de 1998 en el Registro Oficial No. 1; y, concluye con la promulgación de la última Constitución para el Ecuador en octubre de 2008.

En la presente fase, el sistema de educación superior ecuatoriano no es ajeno a la corriente que en el mundo en general se genera con respecto a un modelo de enseñanza basado en competencias, con lo cual se buscaba abandonar el modelo de aprendizaje enciclopédico y formar a los individuos de cara a las exigencias que tiene la sociedad del siglo XXI (Díaz Barriga, 2011).

La Carta Suprema de 1998 establece cambios profundos en el ámbito de la Educación Superior, la Ley de Educación Superior, publicada en el Registro Oficial No. 77, el 15 de mayo del 2000, integra a los institutos técnicos y tecnológicos de las diferentes especializaciones, como parte del Sistema de Educación Superior, esta integración señalada en la Ley, no fue efectiva; pues, mientras se fijaban las funciones para las universidades, la situación de los institutos parecía no preocupar tanto, más aún cuando se confió al Ministerio de Educación el control de los institutos, con lo cual los institutos públicos dependerían financiera y administrativamente del Ministerio de Educación y académicamente del CONESUP.

En lo que respecta a la población estudiantil universitaria encontramos que para el año 2000 ésta se ubicaba en 321.000 estudiantes, crecimiento debido -entre otros factores- al incremento poblacional del país y a la creciente expansión de la educación media (Rojas Pazmiño, 2003).

Se reconoce a la investigación científica como una de las finalidades de las universidades y escuelas politécnicas y el escalafón del docente universitario y politécnico, además, esta Constitución destaca el rol del Estado en el fomento de la ciencia y la tecnología en todos los niveles educativos, dirigidas a mejorar la productividad, la competitividad, el manejo sustentable de los recursos naturales y a satisfacer las necesidades básicas de la población.

Se ratifica la inviolabilidad del recinto universitario, garantizando además el Estado, la igualdad de oportunidades de acceso a la educación superior para quienes cumplan los requisitos establecidos por el sistema nacional obligatorio de admisión y nivelación.

Como una acción significativa en esta fase encontramos el establecimiento de la rendición social de cuentas para las instituciones de educación superior, para lo cual se estableció un sistema autónomo de evaluación y acreditación, que funcionó en forma independiente, en cooperación y coordinación con el Consejo Nacional de Educación Superior (CONESUP). Este organismo, identificado como el Consejo Nacional de Evaluación y Acreditación de la Educación Superior (CONEA), estableció la normativa y diseñó los instrumentos técnicos a través de los cuales debían evaluarse las instituciones del Sistema de Educación Superior del Ecuador, el modelo a aplicar fue definido en base a la medición de la calidad a través de funciones, ámbitos, características, estándares e indicadores. Las funciones objeto de evaluación fueron: Gestión administrativa, docencia, investigación y vinculación con la comunidad.

Sin embargo a lo que estaba prescrito en la Constitución y en la propia Ley de Educación Superior, el control a las instituciones del sistema no demostró los cambios que eran necesarios para lograr la formación integral del ser humano que permita alcanzar la justicia social, que sean capaces de producir propuestas para buscar soluciones a los problemas existentes en un país en donde la educación superior estaba condicionada en gran medida por el origen socio-económico de sus estudiantes, como lo demuestra el estudio realizado bajo el título “Incidencia Distributiva del Gasto Público y Funciones de Demanda en el Ecuador (Younger, Villafuerte, \& Jara, 1997), en donde se señala: "En este estudio se evidencia la inadecuada distribución del gasto en la educación superior, ya que en realidad el gasto estatal no se dirige a los que más lo necesitan ( ... )", refiriéndose a la aplicación de 
la curva de Lorenz ${ }^{6}$ en la distribución del gasto en la educación superior, como parte de su investigación.

Esta fase es reconocida por lo tanto, como la más crítica para la educación superior del Ecuador, cuando las estadísticas marcan un crecimiento del $58 \%$ en el inventario de universidades, este incremento está representado de la siguiente manera: $12 \%$ de las universidades públicas y $46 \%$ de las universidades autofinanciadas. La presencia del mayor número de universidades auto-financiadas están localizadas en la región sierra y en la provincia del Pichincha.

\section{SEXTA FASE:}

\section{HACIA LA REVOLUCIÓN DEL SISTEMA DE EDUCACIÓN SUPERIOR PARA EL BUEN VIVIR}

Con el advenimiento de la última Constitución Política del Ecuador promulgada el 20 de octubre de 2008, vigente hasta la fecha, se da inicio a una nueva etapa para la educación superior en el Ecuador.

Incluso antes de la promulgación de esta nueva Carta Magna, la Asamblea Nacional reunida en Montecristi, Manabí, ante la crítica situación en la que se desenvolvía la educación superior, al amparo del Mandato 14, emitido el 22 de julio de 2008, decide hacer cumplir lo que ya estaba prescrito en la Ley de Educación Superior (LES) del 15 de mayo del año 2000 , con lo cual dispone que se realice una evaluación del desempeño institucional de las universidades y escuelas politécnicas del Ecuador; así como, a los institutos técnicos y tecnológicos que eran parte del sistema, a fin de garantizar calidad, propiciando su depuración y mejoramiento. Esta evaluación fue realizada por separado para los dos grupos de Instituciones de Educación Superior (IES).

Para el caso de las universidades, dicha evaluación se cumplió por parte del CONEA entre junio y octubre de 2009, y las conclusiones y recomendaciones ahí establecidas fueron puestas a consideración ante la Asamblea Constituyente en noviembre del mismo año. Los criterios empleados en esta evaluación fueron: academia, estudiantes y entorno de aprendizaje, investigación y gestión administrativa.
La necesidad de conocer el estado real de cada institución del sistema, hizo que la comisión conformara conjuntos de instituciones con un nivel relativamente homogéneo de desempeño y homogeneidad, en base a lo cual se ubicaron a las 68 universidades en cinco categorías.

En la Categoría A, se ubicaron a las universidades que contaban con estatutos y reglamentos en los cuales estaban claramente definidos los derechos docentes, representada por una planta docente con un sentido de pertenencia a una comunidad universitaria y comprometida, en cierto grado, con el entorno social a través de acciones y programas de vinculación con la colectividad, también se evidenció que estas IES contaban con soporte académico para los procesos de aprendizaje acordes a las exigencias de los avances del conocimiento y además, mostraron un desempeño notable en actividades de investigación.

La ubicación de las universidades en la Categoría $B$, se hizo tomando en cuenta a aquellas en las cuales los deberes y derechos docentes, aunque reglamentados y explícitos en los estatutos y reglamentos, no eran aplicados a plenitud o tendían a ser practicados de manera discrecional. Asimismo, el nivel académico de la planta docente (con una excepción) y su modalidad de dedicación, en general, estaban por debajo de las exigencias de la Ley Orgánica de Educación Superior (LOES). El desarrollo de actividades de investigación se limitaba a proyectos débilmente articulados que no lograban insertarse en las estructuras académicas de las IES.

En la Categoría C, se ubicaron a las IES en las cuales el nivel académico de la planta docente, estaba compuesto en su mayoría por docentes con cursos de diplomado o especialidad, y en los casos que poseían maestrías, éstas no correspondían a la especialidad de la cátedra y, en repetidos casos, habían sido obtenidas en la propia universidad.

Se verificó además, deficiencia en el tiempo de dedicación a las labores docentes; puesto que las IES de este grupo contaban con una planta relativamente significativa de docentes a tiempo completo, la natu-

6 Curva que relaciona el porcentaje acumulado de la población, con el porcentaje acumulado del ingreso que posee esa población. Esta curva permite representar gráficamente la concentración del ingreso de una región en un período determinado. 
raleza de su carga horaria no les permitía dedicarse a actividades de soporte a los estudiantes, ni a otras actividades académicas.

En lo que respecta a la Categoría $\mathrm{D}$, se ubicaron las IES que tenían un alto porcentaje de la planta docente conformada por profesores contratados por horas de clase, con escaso compromiso académico fuera de la obligación de dictar las clases para las que habían sido contratados con una evidente sobrecarga horaria. Además los niveles de remuneración, en muchos casos, eran, sin estabilidad, inexistencia de investigación relacionada por lo menos con la propia docencia, en donde el entorno de aprendizaje y las políticas de acción afirmativa y de vinculación con la colectividad eran bastante heterogéneos; en algunos casos satisfactorio, en otros, con serias deficiencias.

Por último, en la Categoría E se ubicaron a las universidades que no contaban con una comunidad docente estable, académicamente preparada, con obligaciones y derechos reconocidos y con la dedicación necesaria para garantizar una docencia de calidad.

En síntesis se trataba de instituciones que, definitivamente, no presentaban las condiciones que exigía el funcionamiento de una institución universitaria y en las que se evidenciaban las deficiencias y problemas que afectaban a la universidad ecuatoriana.

La clasificación realizada, consta en el gráfico $\mathrm{N}^{\circ} 1$, en donde se demuestra que el mayor porcentaje corresponde a las universidades ubicadas en la Categoría E. De las 26 universidades incluidas en esta categoría, 24 son privadas y sólo dos (02) públicas, señalándose además, que todas estas universidades habían sido creadas en los últimos 12 años; y, 14 de ellas, a partir de la entrada en vigencia de la Ley Orgánica de Educación Superior expedida en mayo de 2000; por lo que el CONEA recomendó la suspensión de estas 26 universidades, evidenciándose de esta manera, la crisis en la que estaba envuelto el Sistema de Educación Superior Ecuatoriano, en relación a las universidades, quedando por comprobarse aún, cuál sería la situación del otro grupo de instituciones del Sistema: los institutos superiores técnicos y tecnológicos.

\section{Gráfico No 01:}

Evaluación de las universidades ecuatorianas según el Mandato 14

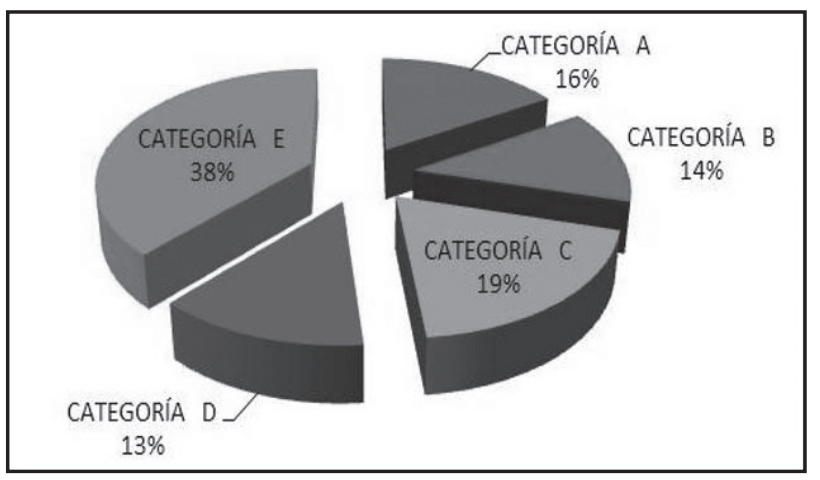

Fuente: Informe CONEA - 2009. Elaboración propia.

La comisión encargada de la evaluación de los institutos superiores técnicos y tecnológicos por su parte, presentó el informe en octubre de 2009, con respecto a la situación en la que se encontraban 285 institutos en funcionamiento al momento de la evaluación. Para esta evaluación, la comisión desarrolló su trabajo en base a tres criterios: docentes, estudiantes y administración, con este soporte se definieron los respectivos sub-criterios e indicadores, con los cuales el resultado de la evaluación a los institutos concluyó en la ubicación de tres categorías, pero la comisión resaltó en su informe que en el criterio docentes se evidenció una débil carrera docente; por lo que es necesario que se fortalezca la misma, a través de la integración de las siguientes variables: sistemas de ingreso, de valuación, de remuneraciones, de promociones, de beneficios, de condiciones de trabajo de capacitaciones científicas y pedagógicas y de retiro.

En lo que respecta a la variable estudiantes es insuficiente la gestión de recursos para la provisión de equipos de laboratorios, talleres, títulos actualizados para la biblioteca y el espacio de lectura para los estudiantes, $\mathrm{o}$ acceso a bibliotecas virtuales. Mientras que, en el criterio administración se evidenciaron limitados servicios de orientación profesional y la oferta de becas a los estudiantes, una infraestructura insuficiente en materia de aulas y equipamiento, maquinaria de los talleres desactualizada para los procesos de aprendizaje, en síntesis falta de gestión de los recursos financieros para mejorar los presupuestos institucionales. 
Tabla No 01

Categorización de los Institutos Superiores - Año 2009

\begin{tabular}{|c|c|c|c|c|c|c|}
\hline \multirow{2}{*}{ TIPO DE INSTITUTO } & \multicolumn{2}{|c|}{ CATEGORÍA A } & \multicolumn{2}{c|}{ CATEGORÍA B } & \multicolumn{2}{c|}{ CATEGORÍA C } \\
\cline { 2 - 7 } & Núm. & $\%$ & Núm. & $\%$ & Núm. & $\%$ \\
\hline Industriales & 30 & 61 & 41 & 22 & 14 & 27 \\
\hline Agropecuarios & 14 & 29 & 03 & 2 & 02 & 04 \\
\hline Administrativos & & & 110 & 59 & 32 & 63 \\
\hline Pedagógicos & 01 & 2 & 29 & 15 & & \\
\hline Conservatorios & 02 & 4 & 04 & 2 & 03 & 6 \\
\hline Arte & 02 & 4 & & & & \\
\hline Totales & 49 & $100 \%$ & 187 & $100 \%$ & 51 & $100 \%$ \\
\hline
\end{tabular}

Elaboración: Propia.

Así, entonces la categorización de los institutos por el grado de cumplimiento, se demuestra en la Tabla $\mathrm{N}^{\circ} 01$.

Es de destacar que el resultado de la evaluación a los Institutos Técnicos y Tecnológicos no creó mayor impacto en la sociedad ecuatoriana, debido a que el funcionamiento de este grupo de instituciones, no pasaba de ser en la mayoría de los casos una extensión del bachillerato, no existiendo incluso vinculación alguna con las universidades, salvo aquellas que por su propia estructura empresarial (sector privado), mantenían a los dos grupos.

Con este diagnóstico del Sistema de Educación Superior del Ecuador, nace el 12 de octubre de 2010 la nueva Ley Orgánica de Educación Superior (LOES), al amparo de la Constitución de 2008 promulgada en Montecristi, que está articulada al Sistema Nacional de Educación y al Plan Nacional de Desarrollo, con lo cual se abre el espacio para la transformación agresiva de la educación superior, reconociendo su autonomía ejercida y comprendida de manera solidaria y responsable, en lo relacionado principalmente a la gestión interna, manejo de presupuesto, administración de patrimonio y recursos y el cogobierno entre profesores, estudiantes, empleados y trabajadores, pero aclarando que esta no exime a las instituciones de ser fiscalizadas, de la responsabilidad social, rendición de cuentas y participación en la planificación nacional. También se regulan los principios de igualdad de oportunidades, la calidad, la pertinencia, la integralidad para todos los actores del sistema de educación superior.
La LOES, en su Disposición Transitoria Tercera, retomó el informe del CONEA, y estableció que las 26 universidades y escuelas politécnicas ubicadas en la categoría "E" debían ser nuevamente evaluadas dentro de un plazo de 18 meses posteriores a la promulgación de la Ley. Las 26 universidades y escuelas politécnicas categoría "E" que no superasen la evaluación del CEAACES debían ser suspendidas definitivamente.

Un aspecto importante que marca la Ley del 2010 es el reconocimiento de la gratuidad de la educación superior pública hasta el tercer nivel, al que tendrán derecho los estudiantes que demuestren responsabilidad académica.

La nueva estructura para el Sistema de Educación Superior (SES) ecuatoriano incluye en primer lugar, a las instituciones educativas del sistema: las universidades, escuelas politécnicas públicas y particulares, los institutos superiores técnicos, tecnológicos, pedagógicos, de artes y los conservatorios superiores, tanto públicos como particulares, los organismos que rigen actualmente el Sistema de Educación Superior son:

La Secretaría Nacional de Educación Superior, Ciencia, Tecnología e Innovación (SENESCYT), Consejo de Educación Superior (CES) y por último, el Consejo de Evaluación y Acreditación y Aseguramiento de la Calidad de la Educación Superior (CEAACES).

Con la vigencia de esta nueva Ley, luego de los 18 meses que estipula la LOES para que las IES que 
Laura Amarilis Borja Herrera - Ena Maritza Feijoo González

Néstor Daniel Gutierréz Jaramillo - Raquel Magali Jaramillo Simbaña

Milca NaAra Orellana Ulloa - Beatriz Herrera García

fueron ubicadas en Categoría "E", pudieran corregir los errores detectados en la evaluación del 2009 por el CONEA, el Consejo de Evaluación, Acreditación y Aseguramiento de la Calidad de la Educación Superior (CEAACES), el 12 de abril de 2012 presenta el informe final de evaluación de las 26 universidades, el mismo que fue elaborado en cumplimiento a la Disposición Transitoria Tercera de la LOES, con lo cual tres (03) universidades fueron clasificadas como ACEPTABLES; ocho (8) PARCIALMENTE ACEPTABLES; $y$ catorce (14) como NO ACEPTABLES.

Considerando que el cierre de estas universidades traía consigo un gran efecto social, toda vez que, la población estudiantil afectada representaba un universo de 42.417 estudiantes, de los cuales 37.080 , que representan el $87 \%$ de los estudiantes que pasaron de la fase de inscripción a la de matriculación, se involucraron en el Plan de contingencia aprobado por el CES como herramienta orientada a garantizar la continuidad de estudios de los estudiantes de las universidades y Escuelas Politécnicas categoría E que fueron suspendidas definitivamente. Este Plan, aprobado por el CES, representa para el Estado una inversión de USD\$ 9.933.830 para el periodo 20122017 , en que se asume que los estudiantes que cursaban sus estudios en estas universidades, concluirían los mismos (Consejo de Educación Superior, 2013).

El proceso de evaluación a las universidades y escuelas politécnicas categoría "E” se ejecutó con la aplicación de un nuevo modelo de evaluación constituido por dos componentes: el entorno de aprendizaje y los resultados de aprendizaje.

En lo que respecta a la situación de los institutos técnicos y tecnológicos, el CEAACES, en sesión del 7 de octubre de 2011, dispuso la suspensión definitiva de 125 institutos de los 407 que figuraban registrados (de los cuales únicamente 285 fueron evaluados en el 2009, porque los otros fueron considerados históricos o sin funcionar y otros que habían dejado de funcionar por diversos motivos).

Pero, el proceso de depuración del sistema de educación superior no concluiría ahí; pues en noviembre de 2013, en cumplimiento a lo dispuesto en la disposición transitoria vigésima de la Constitución del 2008, que establecía que "en el plazo de cinco años, desde la entrada en vigencia de esta Constitución, todas las instituciones de educación superior, así como sus carreras, programas y posgrados deberán ser evaluados conforme a la ley.

En caso de no superar la evaluación y acreditación quedarán fuera del sistema de educación superior", ratificada en la Disposición Transitoria Primera de la LOES expedida en el 2010, el CEAACES, señaló además, que las universidades que son ubicadas en las categorías A, B y C están acreditadas dentro del Sistema de Educación Superior del Ecuador, mientras que las que se ubiquen en la Categoría $\mathrm{D}$, deberán cumplir con un plan de fortalecimiento institucional que les permita ubicarse por lo menos en la categoría C. La Comisión de Evaluación designada por el CEAACES, presenta los resultados que se muestran en el Gráfico $\mathrm{N}^{\circ} 02$, con lo cual, a partir de esta fecha (noviembre de 2013), las IES ubicadas en la Categoría D, disponen de 18 meses, esto es hasta mayo de 2015 para lograr su ubicación en una de las tres categorías que les permita, seguir siendo parte del sistema, para lo cual el CEAACES, les brinda la asesoría necesaria y realiza los monitoreos periódicos, quedando bajo absoluta responsabilidad de la respectiva comunidad universitaria los resultados a obtener.

Por su parte las universidades que se ubicaron en las tres primeras categorías, deben desarrollar sus planes de mejoramiento; pues, la meta trazada es que en el Ecuador sólo existan las dos primeras categorías de universidades.

Pero, la política del gobierno declarado como el Gobierno de la revolución ciudadana, en materia de educación superior, va mucho más allá que la evalua-

Gráfico No 02:

Evaluación de CEAACES 2013

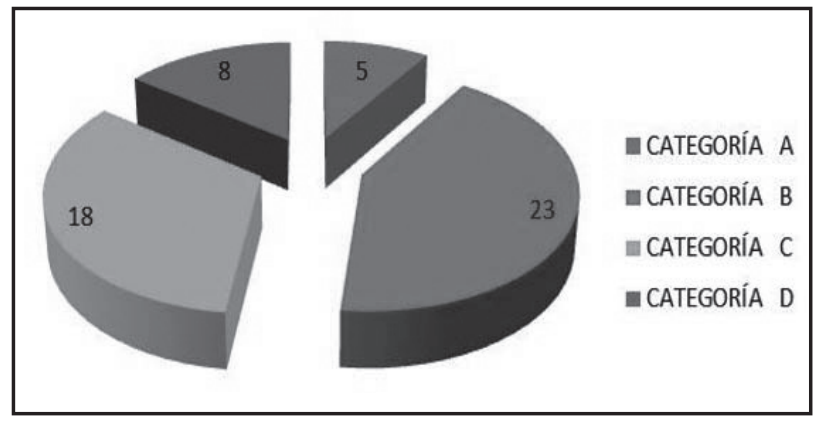

Fuente: CEAACES. Elaboración propia. 
ción de las IES, el objetivo es fortalecer la educación superior a través de acciones emergentes que ya están en marcha:

\section{a. Incremento de inversión en Educación Superior}

Actualmente, el Ecuador invierte el $2.0 \%$ de su PIB en Educación Superior ubicándonos en una posición muy alta en relación a otros países de América Latina, es importante destacar que en promedios establecidos por la OECD (Organización para la Cooperación y el Desarrollo Económicos) los países más ricos invierten el 1,70\%, mientras que los países del sur de América Latina como; Perú, Argentina, Paraguay, Chile, Brasil, Colombia promedian una inversión del 0,90\% del PIB de acuerdo a sus economías.

\section{Gráfico No 03:}

Inversión histórica en educación superior

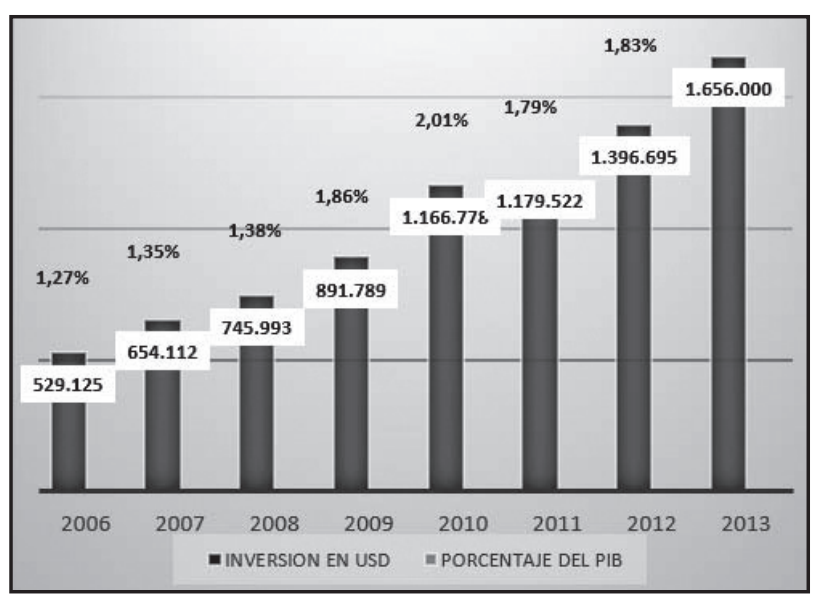

Fuente: SENESCYT. Elaboración propia.

En el gobierno actual denominado "El Gobierno de la Revolución Ciudadana" se ha invertido USD $\$ 8.220$ millones de dólares desde 2006 hasta el periodo 2013 (Gráfico $\mathrm{N}^{\circ}$ 03), convirtiéndose en una inversión histórica para este sector, destacándose que es en el año 2010 en donde la inversión en relación al PIB alcanza su más alto porcentaje (2.01\%), a manera de referencia, hacemos la comparación con los USD \$ 185 millones y USD \$ 240 millones, asignados para los años 2002 y 2003, respectivamente (Rojas Pazmiño, 2003).
Ecuador siempre ha invertido en educación. Sin embargo, durante años no se evidenciaron ni se midieron los resultados como se realiza actualmente.

A partir de la reforma a la Ley de Educación Superior se empiezan a ejecutar un sinnúmero de acciones para eliminar las barreras en el sector educativo superior mediante una reingeniería para reducir el gasto administrativo y el ahorro destinarlo a la mejora académica. Se han tomado acciones como la expedición del reglamento de aplicación de la fórmula de distribución de recursos destinados anualmente por el Estado a favor de las Instituciones de Educación Superior (Consejo de Educación Superior, 2013), en el cual se identifican las respectivas fuentes fijas de ingresos para la educación superior ecuatoriana.

Pero la misma fuente normativa, establece los criterios y los parámetros de distribución y la fórmula de distribución de esos recursos, señalándose que los criterios y parámetros de distribución de los recursos se definen y valoran en base a la calidad, excelencia académica y eficiencia, con lo cual se busca garantizar el cumplimiento de los criterios de equidad y justicia, basados en la calidad, excelencia académica y eficiencia.

Esta distribución dependerá además de la categorización que tenga cada IES, según el último informe CEAACES 2013.

\section{b. Incremento en los salarios de los docentes}

La valoración del docente universitario en términos de justicia y equidad en cuanto a las remuneraciones que percibía evidenciadas en el Informe del CONEA 2009, ha sido una de las mayores preocupaciones del Gobierno actual, especialmente para las universidades que se ubicaron en las categorías C y D, estableciéndose que todavía en el Informe del CEAACES 2012, mantenían esta situación; es para el año 2013, que se identificaron cambios que involucraron una mejora salarial: cuando un profesor auxiliar ganó 2.48 veces más, un profesor agregado recibe 3,12 veces más y un principal ha tenido in incremento del 1,32 veces más en relación a periodos anteriores al año 2012, conforme se demuestra con el Gráfico № 04. 
Laura Amarilis Borja Herrera - Ena Maritza Feijoo González

Néstor Daniel Gutierréz Jaramillo - Raquel Magali Jaramillo Simbaña

Milca NaAra Orellana Ulloa - Beatriz Herrera García

\section{Gráfico No 04:}

Comparación de los salarios docentes antes y luego de la reforma expresada en dólares

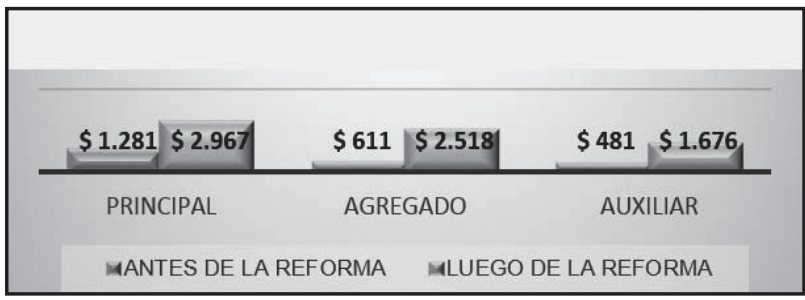

Fuente: SENESCYT. Elaboración propia.

\section{c. Se logra incrementar las becas a estudiantes}

Otro de los grandes cambios observados en este período, es el apoyo entregado por el Gobierno para que los sectores tradicionalmente marginados puedan tener acceso a la educación superior, a través de becas incluso en el exterior, lo que queda demostrado con la concesión de 7.168 créditos no reembolsables para estudios de cuarto nivel en el extranjero, inversión que representa el 47,25\% más en relación a los períodos 1996-2006 (Gráfico No 05).

\section{Gráfico No 05:}

Número de becarios $4^{\circ}$ nivel en el extranjero por recursos propios expresado en dólares

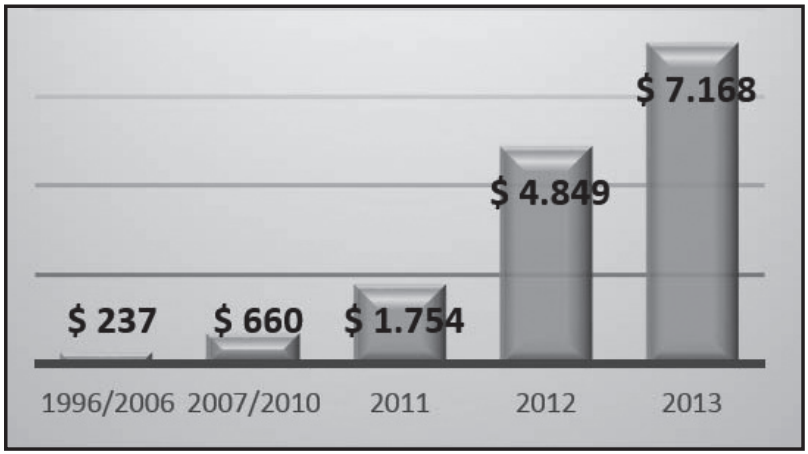

Fuente: SENESCYT. Elaboración propia.

\section{d. Desarrollo de la investigación científica en las universidades del país}

Reconociendo que una de las mayores deficiencias que tiene la universidad ecuatoriana es la limitada investigación vinculada con los proyectos de desarrollo local, regional y nacional, el Gobierno en el año 2011 emite la Reglamentación para el desarrollo del proyecto Prometeo ${ }^{7}$, ubicándose como instancia rectora del mismo a la Secretaría Nacional de Educación Superior, Ciencia y Tecnología, SENESCYT, teniendo la oportunidad las instituciones públicas o universidades que necesiten el apoyo de un experto con el más alto perfil profesional para el diseño o realización de un proyecto, coordinar con esta Secretaría la colaboración del Prometeo o experto científico.

Las estadísticas de la SENESCYT demuestran que hasta el año 2013, se han traído al país 507 expertos de alto nivel internacional, que provienen de 42 países, ellos son encargados de trabajar en diversas áreas estratégicas del Ecuador, habiendo logrado la ejecución de 295 proyectos, teniendo en carpeta la implementación de 212 nuevos proyectos en distintas áreas.

Con el apoyo de este programa se han capacitado cerca de 44.334 ecuatorianos entre estudiantes, docentes, investigadores y ciudadanía, en general, lo que ha tenido una inmediata incidencia en el ámbito investigativo de las universidades, al determinarse un incremento del número de docentes dedicados a la investigación científica que pasó de 205 en el año 2005 a 1.165 en el año $2012^{8}$; esto se ve reflejado además, en el incremento del número de publicaciones científicas en el Ecuador; toda vez que, de los proyectos que se realizaron en los años 2006-2007 se obtuvieron 3 publicaciones, mientras que en los proyectos ejecutados en el 2008, se realizaron 37 publicaciones?.

Como complemento importante para el desarrollo de la investigación, el Estado ha incrementado la inversión en bases de datos científicos para facilitar el acceso al conocimiento. Del año 2010 al 2013 se dio un incremento que va de USD\$ 1.938 .000 en el 2010 a USD\$ 5.214.606 en el $2013^{10}$.

\section{e. Creación de universidades emblemáticas}

Se crearon 4 Universidades de alto rendimiento en el país, especializadas en áreas estratégicas para

7 El Proyecto Prometeo es una iniciativa del gobierno ecuatoriano, que busca fortalecer la investigación, la docencia y la transferencia de conocimientos en temas especializados, a través de la vinculación de investigadores extranjeros y ecuatorianos residentes en el exterior. 8 Investigación realizada a septiembre del año 2013 por la Subsecretaría de Investigación Científica y el SENESCYT.

9 Íbidem

10 Investigación realizada a septiembre del año 2013 por la Subsecretaría de Investigación Científica y el SENESCYT.

198/ QUIPURAMAYOC / Vol. 22(42) 2014 
la nación: la primera es la Universidad Yachay, conocida como la universidad del conocimiento, su fin es promover la investigación, innovación y producción tecnológica como elemento primordial del desarrollo académico y científico; la segunda es la Universidad Ikiam, identificada como la universidad de la biodiversidad, su fin es fomentar la generación del bio-conocimiento enfocada en el desarrollo de investigación para la explotación de recursos naturales no renovables (minas y petróleos); la tercera es la Universidad de las Artes, conocida como la universidad de la cultura, su fin es desarrollar la oferta de programas educativos en el campo de las artes: cine, audiovisuales, artes escénicas y teatrales; y estudios literarios interculturales, entre otros; y la cuarta es la UNAE, conocida como la universidad de la Educación, su propósito es promover la generación de conocimiento asociado al área de Ciencias de la Educación.

\section{CONCLUSIONES}

1. La Constitución del 2008 reconoce el valor de la educación superior, entregándole al Consejo de Educación Superior (CES) la responsabilidad de ente promotor de los procesos de transformación sobre la base del principio del Buen Vivir.

2. El proceso de depuración, fortalecimiento y mejoramiento de las instituciones de educación superior, representa un compromiso no sólo de la comunidad universitaria, sino también, de la sociedad en general.

3. El retorno de los becarios desde las universidades extranjeras con formación de cuarto nivel, representa una opción para reemplazar a los docentes que masivamente se jubilaron en los años 2012 y 2013.

4. Entre los años 2012 y 2013, el Consejo de Educación Superior como organismo rector de la educación superior del Ecuador ha emitido la normativa pertinente para regular el ejercicio de la docencia universitaria y garantizar el eficiente desarrollo de la academia, entre los que destacan el Reglamento de Carrera y Escalafón del Profesor e Investigador del Sistema de Educción Superior, con lo cual se afianzó la reestructuración general del sistema en busca de la armonización de nomenclatura de títulos que permitirá que hasta el año 2015 se cumpla el proceso de depuración de carreras a fin de facilitar la movilidad estudiantil y asegurar la eficiencia académica de las IES.

5. La inversión realizada por el Estado en el mejoramiento de la Educación Superior ha sido agresiva, sobre todo en el Proyecto Prometeo, considerado como único en la región; por lo que se prevé que el nuevo proceso de evaluación previsto para el 2015, tendrá mayores niveles de exigencia hasta alcanzar el objetivo que en el Ecuador únicamente existan universidades de categorías A y B.

En síntesis se podría decir que en el Ecuador en materia de Educación Superior ha nacido la esperanza para la reconstrucción del país, a partir del conocimiento representado en la academia.

\section{REFERENCIAS BIBLIOGRÁFICAS}

1. ACOSTA Navarro, M. E. (2005). Tendencias pedagógicas contemporáneas. La pedagogía tradicional y el enfoque histórico- cultural. Análisis comparativo. Revista Cubana de Estomatología, 42(1).

2. Asamblea Constituyente del Ecuador (2008) Mandato 14-Informe de la evaluación de desempeño institucional de las universidades y escuelas politécnicas del Ecuador.

3. CEAACES, D. d. (s.f.). El Análisis multicriterio es una herramienta de apoyo en la toma de decisiones durante el proceso de planificación que permite integrar diferentes criterios de acuerdo a la opinión de actores en un solo marco de análisis para dar una visión integral. Obtenido de Ceaaces: http://www.ceaaces.gob.ec

4. Colectivos de autores CEPES Universidad de la Habana. (2000). Tendencias pedagógicas en la realidad educativa actual.

5. Consejo de Educación Superior. (2010). Ley Orgánica de Educación Superior. Quito, Martes 12 de octubre de 2010 -- N²98. Quito, Pichincha, Ecuador.

6. Consejo de Educación Superior. (Marzo de 2013). Aprobar el Informe Técnico de Transferencia de Recursos Públicos a favor de la 
Universidades y Escuelas Politécnicas de derecho privado suspendidas definitivamente por el CEAACES para el año 2013. RPC-SO-12No.110-2013. Quito, Pichincha, Ecuador.

7. Consejo de Educación Superior. (Abril de 2013). Reglamento de Aplicación de la Fórmula de Distribución de Recursos destinados anualmente por parte del Estado a favor de las Instituciones de Educación Superior. RPC-SE04-No.021-2013. Quito, Pichincha, Ecuador.

8. Consejo de Evaluación, Acreditación y Aseguramiento de la Calidad de la Educación Superior. (2013). Recuperado el julio de 2014, de http://www.ceaaces.gob.ec/sitio/wp-content/ uploads $/ 2014 / 02 / \mathrm{m}$.-Mecanismos-de-rendicio\%CC\%81n-de-cuentas-2012

9. DÍAZ Barriga, Á. (2011). Competencias en Educación.Corrientes de pensamiento e implicaciones para el currículo y el trabajo en el aula. Revista Iberoamericana de Educación Superior (RIES), II(5).

10. ICESI. (s.f.). ICESI.EDU.CO. Recuperado julio de 2014, de CURVA-LORENZ: http://www. icesi.edu.co/cienfi/images/stories/pdf/glosario/curva-lorenz.pdf
11. JAMIL Salmi, “. S. (s.f.). WORDBANK. Obtenido de www1.wordbank.org/education/tertiary

12. OCDE, El Banco Mundial "Evaluación de las políticas nacionales de educación superior en Colombia” (2011) La educación superior en Colombia.

13. RAMÍREZ, R., \& Analía, M. (2009). Transformaciones en la educacòn superior ecuatoriana: Antecedentes y perspectivas futuras como consecuencia de la nueva Constitución Política.

14. ROJAS Pazmiño, J. (2003). Antecedentes, Situación actual y perspectivas de la evaluación y la acreditación de la educación superior. Consejo Nacional de Evaluación y Acreditación UNESCO/IESALC.

15. YOUNGER, S., Villafuerte, M., \& Jara, L. (1997). Incidencia distribuida del gasto público y funciones de demanda en el Ecuador. Recuperado julio de 2014, de FLACSO: https:/ / www.flacso. org.ec $/$ biblio $/$ catalog/resGet.php?resId=14941 